\title{
Modelo de Neurônio Memristivo de Dióxido de Vanádio com Codificação de Impulsos Sigma-Delta-PWM
}

\author{
Bruno Aparecido Sousa Figueiredo Sena* \\ Luiz Alberto Luz de Almeida** \\ * Universidade Federal do ABC, SP, (e-mail: \\ bruno.sena@ufabc.edu.br). \\ ** Universidade Federal do ABC, SP, (e-mail: \\ luiz.almeida@ufabc.edu.br)
}

\begin{abstract}
The study of vanadium dioxide $\left(\mathrm{VO}_{2}\right)$ for neuromorphic computing has been of growing interest in recent years. Due to its hysteretic metal-insulation transition, which can be used as memory, $\mathrm{VO}_{2}$ thin films can be used to implement the leaky integrate-and-fire memristive neuron $\left(\mathrm{NMLIFVO}_{2}\right)$. The proposed $\mathrm{NMLIFVO}_{2}$ behaves in a way analogous to the brain spike-based processing, pursuing the goals of neuromorphic computing which are the implementation in small size and low power consumption. The change of conductivity under the action of pulsed signals allows the $\mathrm{NMLIFVO}_{2}$ treat information as an electronic equivalent to a biological neuron with synapses. In this work, we present simulation results of the $\mathrm{NMLIFVO}_{2}$ being excited by different voltage inputs converted into sigma-delta modulation, multiplied by synaptic weights using pulse width modulation. Preliminary results indicate that the proposed neuron is a promising device for neuromorphic spiking neural networks.

Resumo: $\mathrm{O}$ estudo do dióxido de vanádio $\left(\mathrm{VO}_{2}\right)$ em computação neuromórfica tem sido de crescente interesse nos últimos anos devido a sua transição de fase metal-isolante. Esta característica histerética é utilizada neste estudo para implementar o modelo de neurônio memristivo leaky integrate and fire de dióxido de vanádio $\left(\mathrm{NMLIFVO}_{2}\right)$. O NMLIFVO $\mathrm{NM}_{2}$ proposto comporta-se de maneira análoga ao cérebro, visto que também baseia-se em disparos neurais que é uma característica que se alinha com a possibilidade de se atender dois dos principais objetivos da computação neuromórfica, que são a implementação física em pequeno tamanho e baixo consumo de energia. A mudança de condutividade sob a ação de sinais pulsados torna o $\mathrm{NMLIFVO}_{2}$ um análogo eletrônico quase ideal de um neurônio biológico com sinapse. Neste trabalho é apresentado resultados de simulação do $\mathrm{NMLIFVO}_{2}$ sendo excitado por diferentes entradas de tensão convertidas em modulação sigma-delta, multiplicadas por pesos sinápticos usando a modulação por largura de pulso. Resultados preliminares indicam que o neurônio proposto é um dispositivo promissor para redes neurais artificiais neuromórficas.
\end{abstract}

Keywords: Neuromorphic Computing; Spiking Neural Networks; Memristor; Vanadium Dioxide; Leaky Integrate and Fire.

Palavras-chaves: Computação Neuromórfica; Rede Neural Artificial; Memristor; Dióxido de Vanádio; Leaky Integrate and Fire.

\section{INTRODUÇÃO}

\subsection{Principais conceitos}

O Dióxido de Vanádio $\left(\mathrm{VO}_{2}\right)$ é um composto inorgânico de grande relevância na indústria de sensores e transdutores, que têm uma característica particular muito interessante que é o surgimento de histerese na transição entre fases de sua estrutura cristalográfica. $\mathrm{O} \mathrm{VO}_{2}$ pode variar entre a fase metal e a fase isolante, dependendo da temperatura aplicada a este quando em forma de filme fino (Jerominek et al., 1993). Na região de transição deste material ocorre uma drástica mudança na condutividade elétrica, por um fator de até $10^{5}$ vezes em monocristais segundo Zylbersztejn and Mott (1975) e até $10^{3}$ vezes em filmes finos policristalinos como afirmado por Griffiths and Eastwood (1974).

A histerese é uma propriedade apresentada por materiais e sistemas físicos que descreve a capacidade de memória dos mesmos (Visintin, 1994). Segundo Almeida (2003) com o intuito de representar matematicamente a histerese em materiais predispostos à mesma, podem ser utilizados métodos numéricos, preferencialmente de baixo custo computacional e complexidade reduzida. O método numérico $\mathrm{L}^{2} \mathrm{P}$ (Limiting Loop Proximity), representado por uma equação algébrica de quatro parâmetros de entrada, é capaz de retratar, de maneira acertada, a evolução histerética da fração volumétrica de microcristais de $\mathrm{VO}_{2}$, e também a relação histerética da resistência pela temperatura (RxT) 
do mesmo (de Almeida et al., 2001); (de Almeida et al., 2002); (Almeida, 2003)).

Devido ao comportamento memristivo que ocorre no dióxido de vanádio decorrente diretamente da histerese que existe na transição de fase metal-isolante é proposto a utilização do material em computação neuromórfica (Driscoll et al., 2009). O atributo chave de um memristor é o fato de que a resistência é dependente não do valor instantâneo da tensão aplicada como em dispositivos que seguem a lei de Ohm, mas de todo o histórico dinâmico da carga que flui no sistema.

Os dispositivos memristivos em uma arquitetura de computação neuromórfica têm potencial de processamento de dados com maior rapidez, menor consumo de energia e tamanho reduzido (Gupta et al., 2019). Essas características fornecem razões convincentes para o desenvolvimento de hardware que empregue a arquitetura neuromórfica, neste estudo é proposto um dispositivo memristivo baseado no material dióxido de vanádio. Gupta et al. (2019) propôs um dispositivo memristivo com essas características baseadas no material Perovskita.

Redes neurais artificiais memristivas são desejadas por suas possíveis características de tolerância a falhas, confiabilidade diante de erros de hardware, pequeno tamanho, baixo consumo de energia, como também melhores respostas em sistemas on-line (Schuman et al., 2017). Wang et al. (2018) propôs um dispositivo memristor baseado em nanopartículas de prata em um filme dielétrico para criar um neurônio artificial com dinâmica de integração e disparo com vazamento e tempo de integração ajustável, onde também integrou-se esses neurônios com sinapses memristivas não voláteis para construir redes neurais artificiais totalmente memristivas. Com essas redes integradas, o autor demonstrou experimentalmente a atualização não supervisionada de pesos sinápticos e classificações de padrões. O objetivo deste trabalho é a proposta de um neurônio memristivo de dióxido de vanádio para posterior aplicação em rede neural artificial memristiva.

\subsection{Modelo Matemático de Histerese da Transição de fase} Metal-Isolante em Filmes Finos de $\mathrm{VO}_{2}-L^{2} \mathrm{P} T M I \mathrm{VO}_{2}$

Com o intuito de obter uma representação matemática acurada da histerese em filmes finos de dióxido de vanádio, foi proposto por Almeida uma adaptação ao modelo de Preisach (de Almeida et al., 2001); (de Almeida et al., 2002); (Almeida, 2003), que utiliza baixa carência computacional e complexidade matemática reduzida que é o modelo de proximidade ao laço principal (Almeida, 2003). Com a adaptação deste modelo histerético ao comportamento e dados experimentais do comportamento histerético do filme fino de $\mathrm{VO}_{2}$ em sua transição de fase tem-se o que neste trabalho é nomeado de modelo histerético $\mathrm{L}^{2} \mathrm{P}$ TMI $\mathrm{VO}_{2}$ ou em outras palavras a modelagem do dióxido de vanádio na sua região histerética.

O modelo descreve a relação histerética da resistência com a temperatura ( $\mathrm{RxT}$ ) do filme fino de $\mathrm{VO}_{2}$ como também é capaz de retratar, com acurácia, a evolução da fração volumétrica de microcristais em um filme fino de $\mathrm{VO}_{2}$ em função da temperatura de exposição do mesmo (gxT) (de Almeida et al., 2002).
A histerese no $\mathrm{VO}_{2}$ pode ser descrita basicamente utilizandose quatro equações: da temperatura de proximidade no início de cada trajetória $\left(T_{p r}\right)$, a relação da fração volumétrica $(g)$, a função de proximidade $(P(x))$ e a condutância do $\mathrm{VO}_{2}\left(C \frac{d T}{d t}\right)$.

No início de cada trajetória no ponto de reversão $\left(\mathrm{T}_{r}, \mathrm{~g}_{r}\right)$, a temperatura de proximidade que expressa a distância de um ponto de interesse inicial a um ponto correspondente final numa determinada trajetória é denotada por $\mathrm{T}_{p r}$ e é dado por,

$$
T_{p r}=\delta \frac{w}{2}+T_{c}-\frac{1}{\beta} \operatorname{arctanh}\left(2 \mathrm{~g}_{\mathrm{r}}-1\right)-\mathrm{T}_{\mathrm{r}}
$$

A fração volumétrica $(g)$ é dada por,

$$
\begin{aligned}
g= & F(T)=\frac{1}{2}+ \\
& \frac{1}{2} \tanh \beta\left(\delta \frac{\mathrm{w}}{2}+\mathrm{T}_{\mathrm{c}}-\left(\mathrm{T}+\mathrm{T}_{\mathrm{pr}} \mathrm{P}\left(\frac{\mathrm{T}-\mathrm{T}_{\mathrm{r}}}{\mathrm{T}_{\mathrm{pr}}}\right)\right)\right)
\end{aligned}
$$

onde $w$ é largura da histerese, $\beta$ é a inclinação da histerese e está relacionada com $\frac{d g}{d T}$ em $T_{c}$ e $\delta=\operatorname{sgn}(\mathrm{g})$, sinal de g.

$\mathrm{O}$ termo $\mathrm{T}_{c}$ é a temperatura crítica, usualmente definida como a temperatura na qual $\mathrm{g}=0,5$. No caso característico de histerese há dois valores para Tc, um para a curva ascendente principal e outro para a curva descendente principal. É assumido neste trabalho $\mathrm{T}_{c}$ com o valor médio para $\mathrm{g}=0,5$, no centro da curva de histerese.

A função de proximidade está relacionada as propriedade do $\mathrm{VO}_{2}$, e é dada por,

$$
P(x)=\frac{1}{2}(1-\operatorname{sen}(\gamma \mathrm{x}))\left(1+\tanh \left(\pi^{2}-2 \pi \mathrm{x}\right)\right)
$$

onde $\gamma$ é uma constante arbitrária, e $x$ é dado por,

$$
x=\frac{T-T_{r}}{T_{p r}}
$$

Para obter-se o gráfico em relação a resistência $(R)$, sabendo que,

$$
R=g R_{s}+R_{m}
$$

podemos reescrever a equação 5 como,

$$
R(T)=R_{0} \exp \left(\frac{2553}{\mathrm{~T}+273}\right) \mathrm{F}(\mathrm{T})+\mathrm{R}_{\mathrm{m}}
$$

onde $R_{0}$ é a resistência em regime isolante, $R_{m}$ é a resistência em regime metal e $\mathrm{R}_{s}$ é a resistência na região semicondutora.

A equação da condutância em tensão é dada por,

$$
C \frac{d T}{d t}=\frac{V^{2}}{R(t)}-g\left(T-T_{s}\right)
$$

onde $\mathrm{T}_{s}$ é a temperatura do substrato.

Sabendo que $\frac{V^{2}}{R}=P=I^{2} R$, podemos reescrever a função em termos da corrente,

$$
C \frac{d T}{d t}=I^{2} R(t)-g\left(T-T_{s}\right)
$$




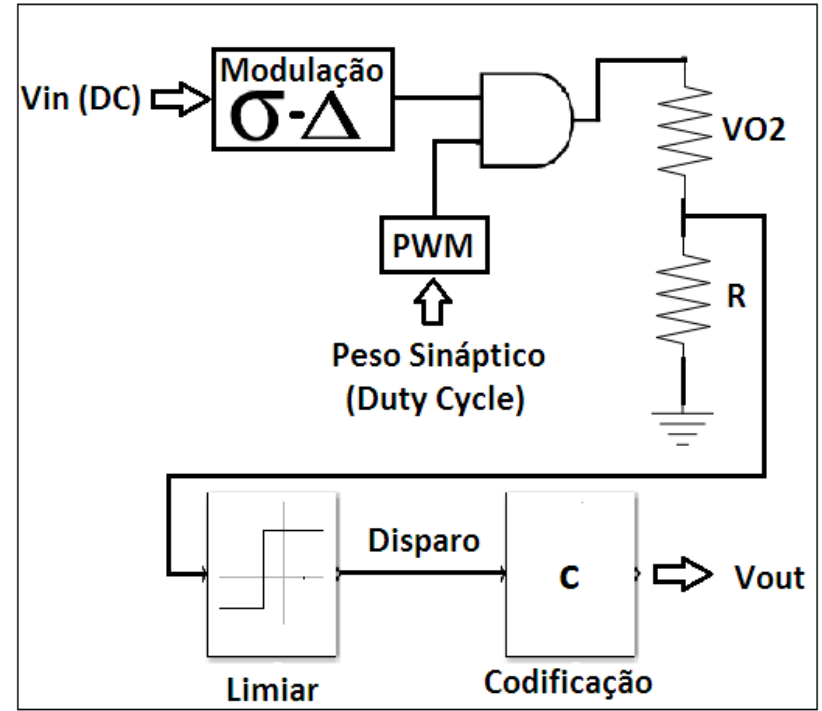

Figura 1. Representação do Neurônio Memristivo leaky integrate and fire de $\mathrm{VO}_{2}\left(\mathrm{NMLIFVO}_{2}\right)$.

\section{METODOLOGIA}

\subsection{Neurônio Memristivo leaky integrate and fire de $\mathrm{VO}_{2}$ $\left(\mathrm{NMLIFVO}_{2}\right)$}

$\mathrm{Na}$ figura 1 está a representação do $\mathrm{NMLIFVO}_{2}$. Vin (DC) é a tensão de entrada que neste trabalho pode assumir valores entre 0 e $1 \mathrm{~V}$, esta entrada é convertida pela modulação sigma-delta e com este sinal modulado é feito uma operação lógica $A N D$ com o sinal em modulação por largura de pulso que pode ter como peso sináptico (Duty Cycle) valores entre $1 \%$ e $100 \%$. A resultante destes sinais é inserida como entrada do neurônio memristivo de dióxido de vanádio, repare que essa resultante pode ser comparada a disparos neurais de tensão, visto que na resultante tem-se valores $0 \mathrm{~V}$ ou $1 \mathrm{~V}$ com intervalos dependentes da entrada e peso sináptico inserido.

É analisado em simulação o comportamento do $\mathrm{NMLIFVO}_{2}$ variando-se o valor de entrada (Vin(DC)) ou o valor do peso sináptico (Duty Cycle). No presente estudo é obtido o comportamento de integração e disparo do neurônio memristivo de dióxido de vanádio com diversos valores de entrada e peso sináptico.

A modulação sigma-delta do projeto converte um sinal de tensão analógica de entrada com valores entre $0 \mathrm{~V}$ e $1 \mathrm{~V}$, com resolução de $0.01 \mathrm{~V}$, em uma frequência de pulsos com amplitude de 1V, exemplo na Figura 2. Com este sinal modulado é feito uma operação lógica $A N D$ com a resultante da modulação por largura de pulso do peso sináptico, sendo que o peso sináptico pode assumir valores entre $1 \%$ e $100 \%$, com resolução de $1 \%$ e amplitude de $1 \mathrm{~V}$, exemplo na Figura 3. Esta resultante final que se assemelha a disparos neurais, mais conhecidos como spikes é a entrada do neurônio $\left(\mathrm{NMLIFVO}_{2}\right)$ que foi modulado e simulado em software MATLAB.

\subsection{Pseudo-código Histerese $L^{2} \mathrm{P} T M I \mathrm{VO}_{2}$}

No algoritmo 1 está o pseudo-código da modelagem e simulação no software MATLAB da histerese $\mathrm{L}^{2} \mathrm{P}$ TMI

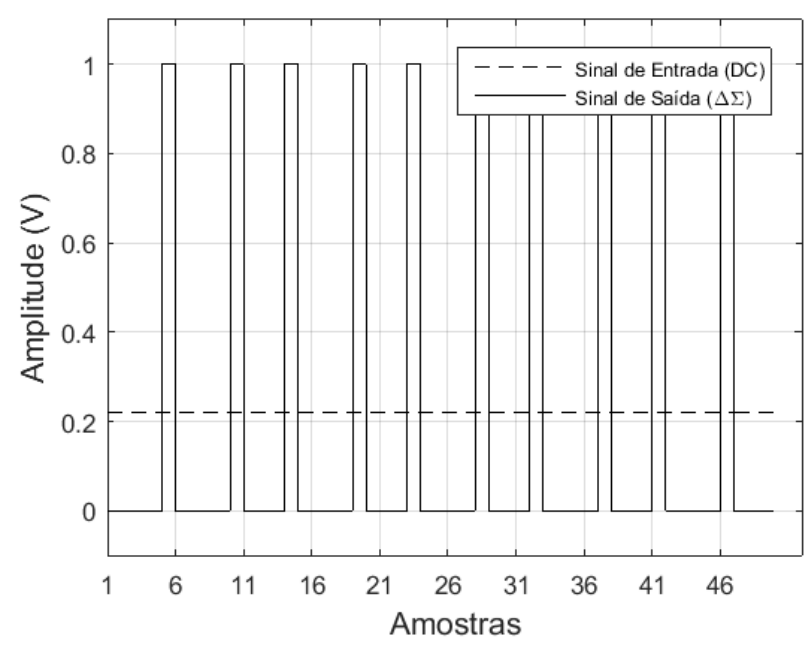

Figura 2. Modulação Sigma-Delta utilizada no projeto exemplo para uma entrada (Vin(DC)) de $0.22 \mathrm{~V}$.

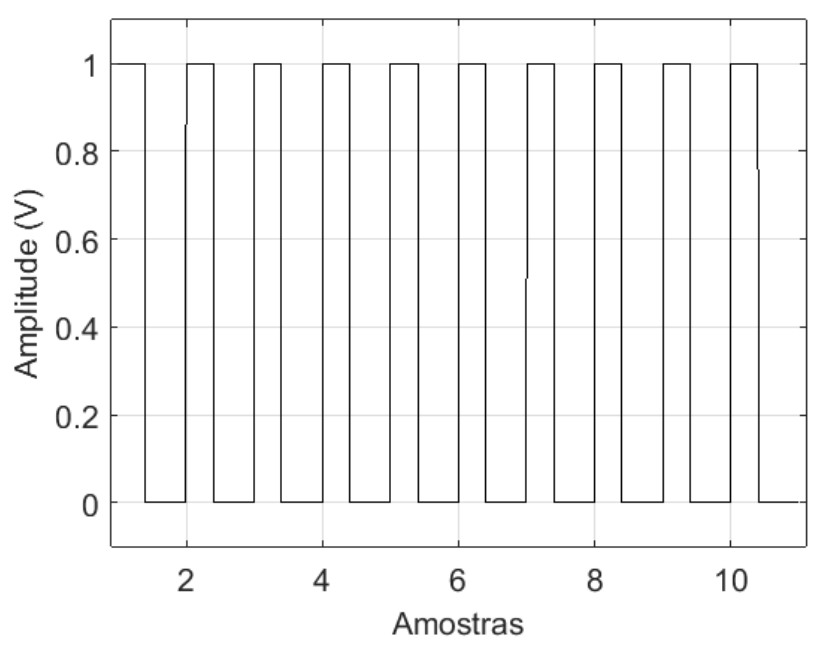

Figura 3. Modulação por largura de pulso utilizado no projeto - exemplo para um peso sináptico (duty cycle) de $40 \%$.

$\mathrm{VO}_{2}$ que é o comportamento do filme fino de $\mathrm{VO}_{2}$ na região de transição de fase. Para a resolução das equações diferenciais ordinárias é utilizado o método de resolução numérica de soluções, Runge-Kutta. Tem-se no projeto a histerese embarcada no Runge-Kutta por causa de possíveis problemas no chaveamento da histerese. Outros métodos mais simples de resoluções de equações não eram confiáveis.

O algoritmo 2 é o algoritmo da função de proximidade que é necessário no algoritmo da histerese $\mathrm{L}^{2} \mathrm{P}$ TMI $\mathrm{VO}_{2}$.

$\mathrm{Na}$ figura 4 a simulação da histerese $\mathrm{L}^{2} \mathrm{P}$ TMI $\mathrm{VO}_{2}$ é apresentada.

\section{RESULTADOS}

Os primeiros resultados são as figuras da modelagem da histerese $\mathrm{L}^{2} \mathrm{P}$ TMI $\mathrm{VO}_{2}$ que são apresentados na metodologia. Essa modelagem do comportamento de um filme fino de $\mathrm{VO}_{2}$ em sua fase de transição é bem relevante, pois pode ser utilizado em várias tentativas de aplicações 
1 Algoritmo 1: Histerese $\mathrm{L}^{2} \mathrm{P}$ TMI $\mathrm{VO}_{2}$

Entrada: $\mathrm{w}, \mathrm{Tc}, \beta, \gamma, \Delta_{a} n, \Delta_{a} t, \mathrm{Tx}, \mathrm{g}$

Saída: Tr, gr, g(1), g(2)

\section{2 início}

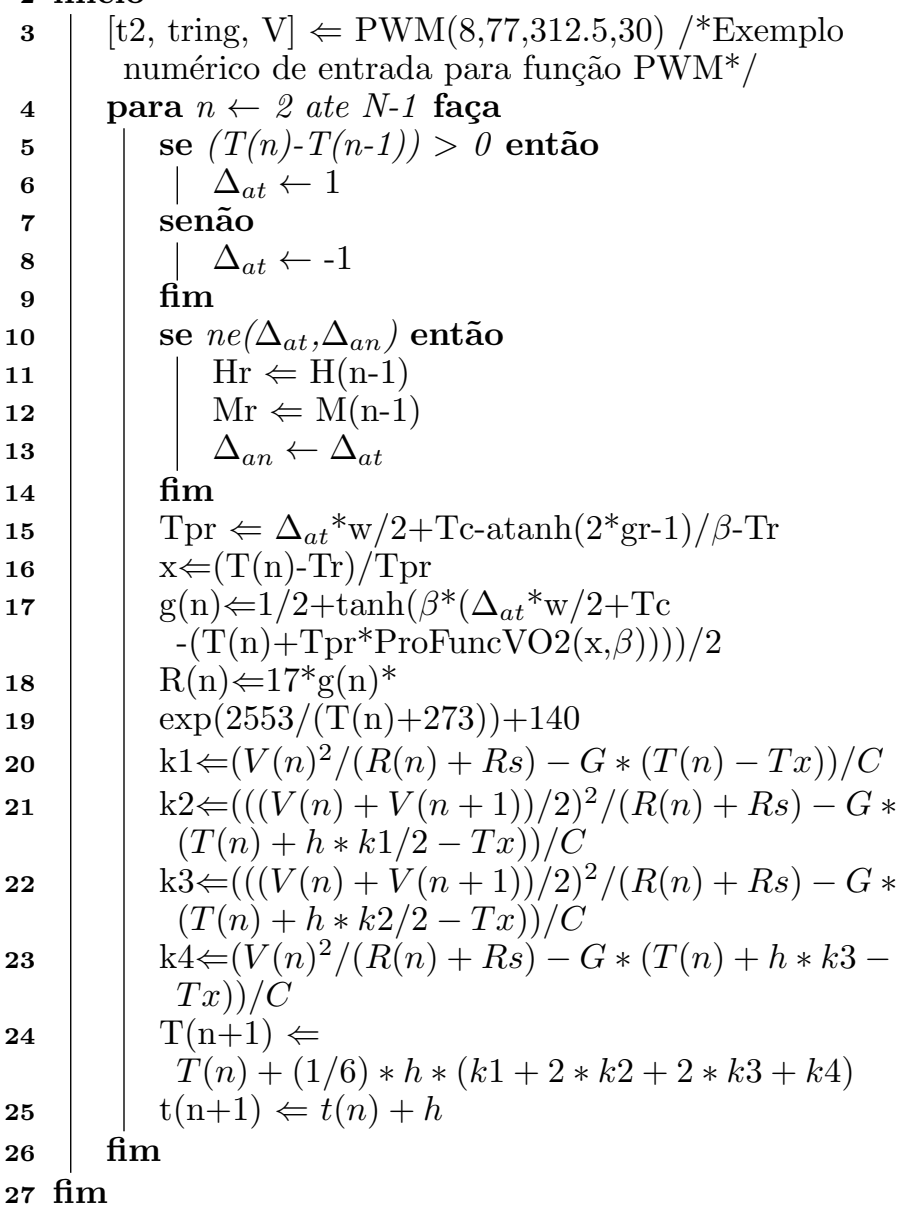

1 Algoritmo 2: Função de Proximidade $\mathrm{L}^{2} \mathrm{P} \mathrm{VO}_{2}$

Saída: y

2 início

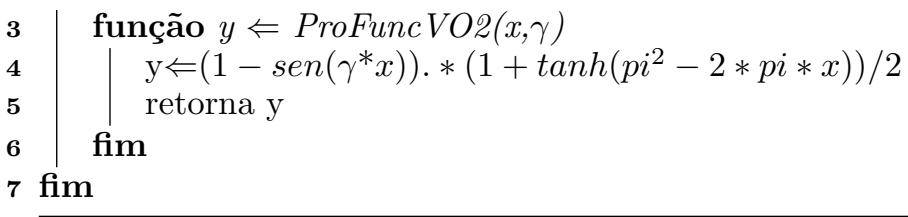

em computação neuromórfica ou em outras áreas, visto que, várias aplicações utilizando das características de transição de fase metal-isolante de diversos matérias estão sendo objeto de estudo mundial e as características e possibilidades de simulações da transição de fase metalisolante do $\mathrm{VO}_{2}$ são bem promissores em várias frentes de estudos.

Aplicações em redes neurais artificiais neuromórficas, ou seja, redes neurais artificiais implementadas com o $\mathrm{NMLIFVO}_{2}$ são os próximos passos deste estudo. Neste artigo é analisado apenas o neurônio $\left(\mathrm{NMLIFVO}_{2}\right)$ que será utilizado na rede neural artificial neuromórfica. Neste neurônio como dito é possível através da entrada de um sinal de tensão num determinado range e "multiplicada"(operação $A N D$ ) por pesos sinápticos que também
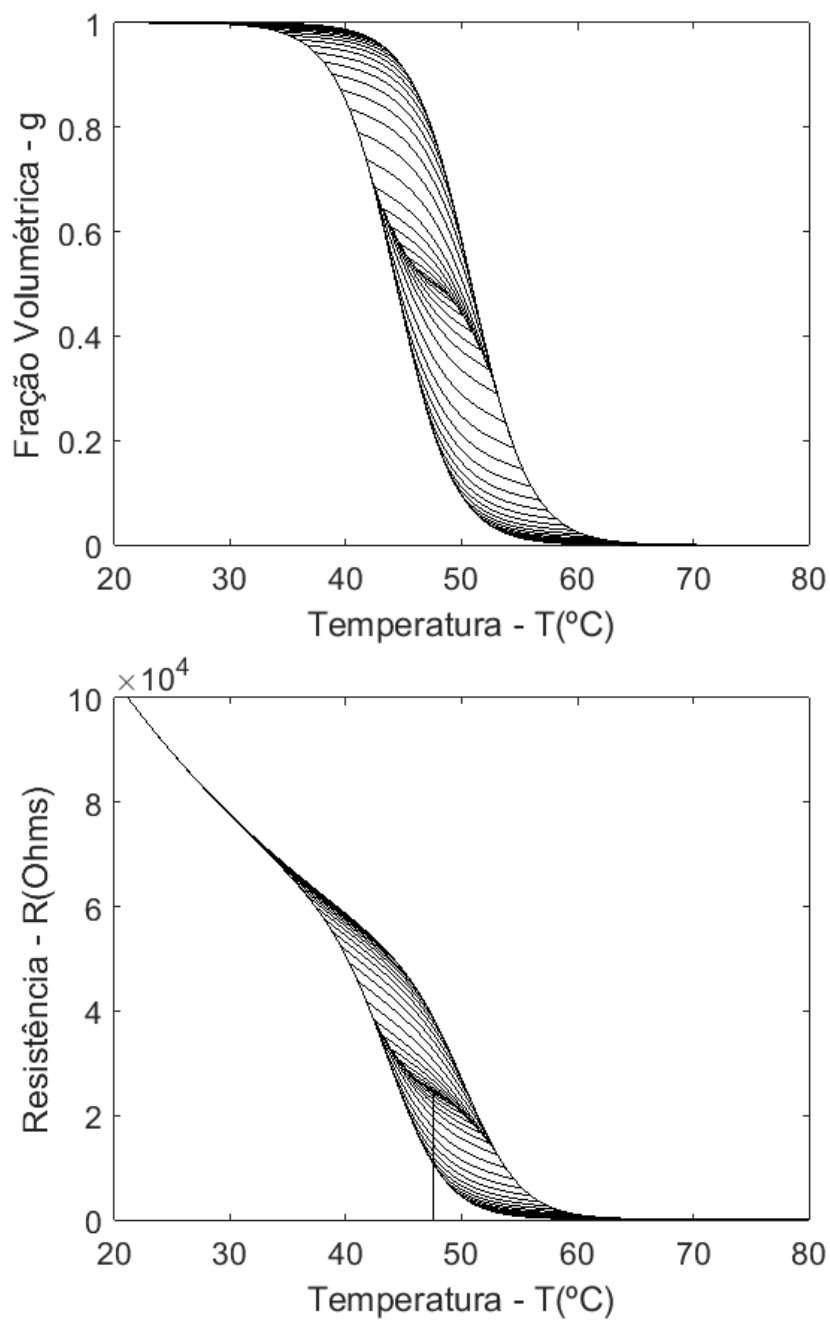

Figura 4. Modelo $\mathrm{L}^{2} \mathrm{P}$ TMI $\mathrm{VO}_{2}$ simulado em software Matlab, parâmetros $\mathrm{w}=6.5, \mathrm{Tc}=47.6$, beta $=0.2$, gama $=0.85$. (a) Curvas características da evolução da fração volumétrica. (b) Curvas características resistência-temperatura.

tem um determinado range (ver Metodologia) que sua resultante gere pulsos comumente chamados de spikes que excitarão nosso neurônio.

Nas figuras 5 e 6 pode-se observar os resultados dessas excitações em temperatura e em resistência. Observando a resposta em resistência pode-se reafirmar como já dito anteriormente que o $\mathrm{NMLIFVO}_{2}$ é um análogo quase ideal de um neurônio biológico com sinapse por sua característica de mudança de condutividade sob ação de sinais pulsados ou em outras palavras sobre a ação de spikes. $\mathrm{O}$ próximo passo é utilizar este neurônio em aplicações práticas de redes neurais artificiais neuromórficas e analisar suas respostas e performance se comparadas com as redes neurais artificiais tradicionais, lembrando que a principal motivação para um sistema neuromórfico ou no caso uma rede neural artificial neuromórfica é a possibilidade de um sistema com consumo baixo de energia, isto é desejável em diversas aplicações. 

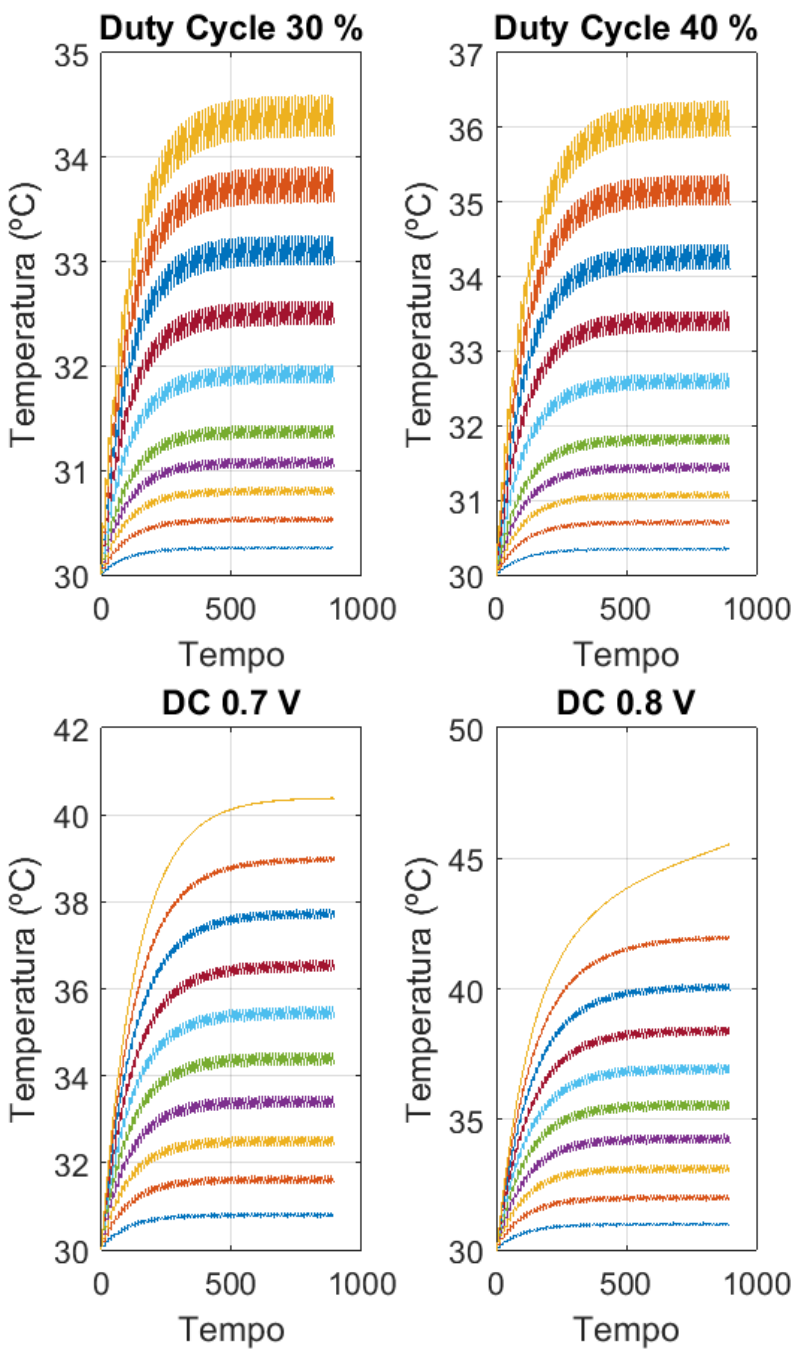

Figura 5. Respostas em temperatura. (a) Peso sináptico (Duty Cycle) fixo e resposta para vários valores de entrada (Vin(DC)) variando de 0.1 até $1.00 \mathrm{~V}$, de $0.1 \mathrm{em}$ $0.1 \mathrm{~V}$, respectivamente, de baixo para cima, primeiro valor de entrada de 0.1V. (b) Entrada (Vin(DC)) fixa e resposta para vários valores de pesos sinápticos (Duty Cycle) variando de 10 até $100 \%$, de 10 em 10\%, respectivamente, de baixo para cima, primeiro peso sináptico de $10 \%$.

Segue um exemplo de como essa rede neural artificial neuromórfica pode funcionar. Atente para a figura $6 \mathrm{~b}$ e suponha que um neurônio foi projetado para ativação em valores menores que $70 \mathrm{~K} \Omega$. Se o peso sináptico desse neurônio estivesse em algum momento fixo em 30\% como o da figura $6 \mathrm{~b}$ a esquerda, apenas entradas iguais ou superiores a $0,7 \mathrm{~V}$ disparariam o neurônio, ou apenas quando esse neurônio recebesse os vários sinais em suas entradas e conseguisse integrar até o valor de $0,7 \mathrm{~V}$ ou superior que esse neurônio iria disparar e assim gerar a entrada para o neurônio da próxima camada.

Na figura 1 está a representação do neurônio, o limiar que está na representação é algo a ser parametrizado para cada neurônio dentro da rede neural artificial neuromórfica (RNAN) com base na análise das figuras 5 e 6, ou seja, cada neurônio dentro da RNAN terá um ponto de ativação/disparo e esse disparo quando ocorrer gerará a entrada
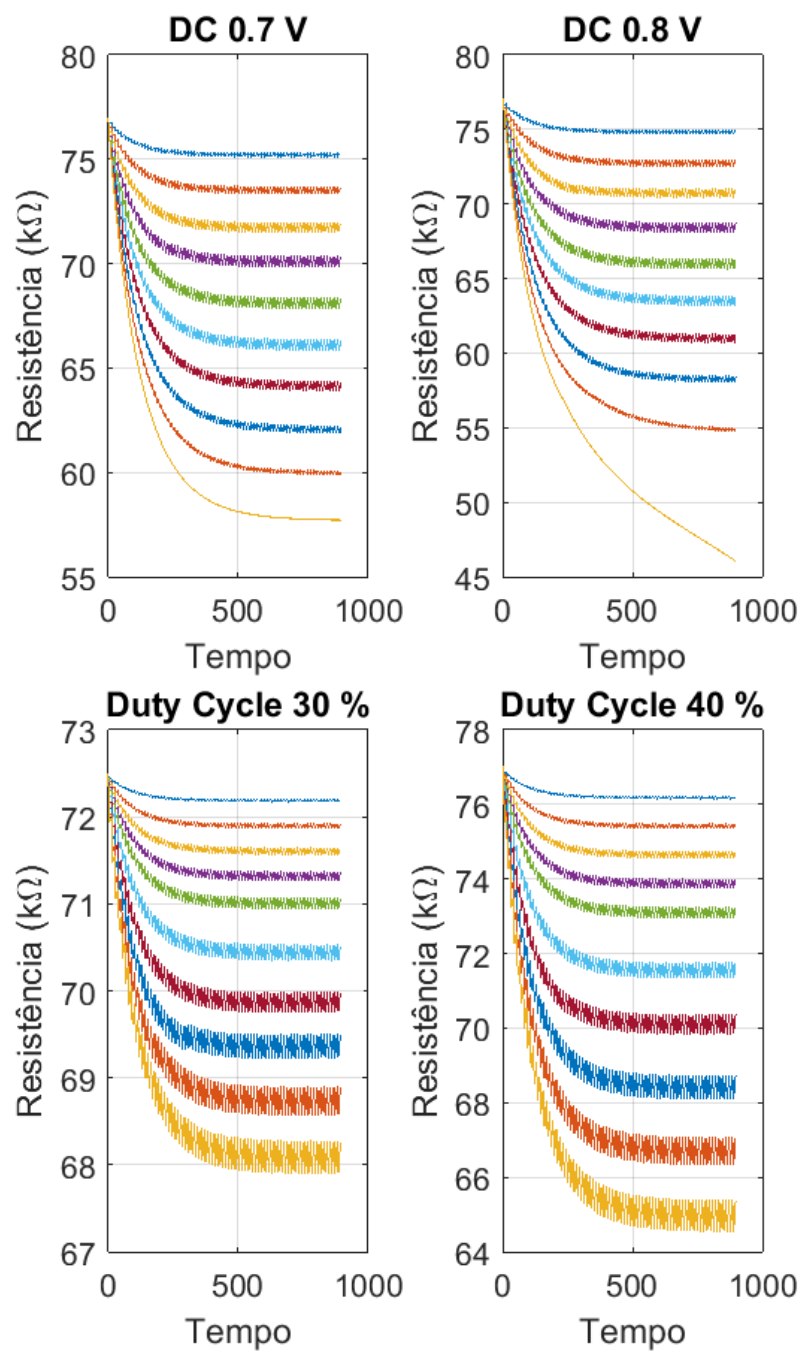

Figura 6. Resposta em resistência. (a) Entrada (Vin(DC)) fixa e resposta para vários valores de peso sináptico (Duty Cycle) variando de 10 até $100 \%$, de 10 em $10 \%$, respectivamente, de cima para baixo, primeiro peso sináptico de $10 \%$. (b) Peso sináptico (Duty Cycle) fixo e resposta para vários valores de entrada (vin(DC)). Variando de 0.1 até $1.00 \mathrm{~V}$, de 0.1 em $0.1 \mathrm{~V}$, respectivamente, de cima para baixo, primeiro valor de entrada de $0.1 \mathrm{~V}$.

dos próximos neurônios conectados a este em formato de uma codificação que também será objeto de estudos posteriores.

Pensando na RNAN que será proposta, quando não se atinge o limiar do neurônio não há disparo da sequência de codificação de pulsos e sendo assim não chegará sinal no próximo neurônio e por causa disso não há comutação desse neurônio e isso faz com que ocorra economia de energia. Cada neurônio só comuta quando o neurônio anterior a este for disparado e isso faz com que haja economia de energia, visto que, quando não há o disparo do neurônio anterior o neurônio atual entra em modo de espera e isso significa que esse neurônio não estará trabalhando. 


\section{CONCLUSÕES}

Foi proposto e simulado com sucesso o neurônio memristivo leaky integrate and fire de dióxido de vanádio e excitado o mesmo com diversos valores de entradas e pesos sinápticos e verificou-se através dos resultados de simulação que seu comportamento está alinhado aos objetivos e comportamentos de sistemas neuromórficos como também é promissor sua utilização em redes neurais artificiais neuromórficas.

Espera-se em trabalhos futuros propor e implementar redes neurais artificiais neuromórficas com este neurônio.

Observa-se na academia o atual e relevante interesse por dispositivos memristores e se atentando para Gupta et al. (2019) e Wang et al. (2018) por exemplo, observa-se que existem vários pesquisadores testando vários materiais para esse propósito e este trabalho agrega como mais uma boa possibilidade de material a ser utilizado como um dispositivo memristor.

\section{AGRADECIMENTOS}

Agradeço a CAPES pelo apoio financeiro via bolsa de estudos.

Agradeço ao Professor Luiz pela dedicação e conhecimentos transmitidos que possibilitaram a realização dos trabalhos.

Agradeço a Deus pelas ajudas diárias.

\section{REFERÊNCIAS}

Almeida, L. (2003). Modelo de histerese para transição semicondutor-metal em filmes finos de vo2. Faculdade de Engenharia Elétrica e da Universidade Estadual de Campinas Grande.

de Almeida, L.A.L., Deep, G.S., Lima, A.M.N., Neff, H.F., and Freire, R.C.S. (2001). A hysteresis model for a vanadium dioxide transition-edge microbolometer. IEEE Transactions on Instrumentation and Measurement, 50(4), 1030-1035.

de Almeida, L.A.L., Deep, G.S., Nogueira-Lima, A.M., and Neff, H. (2002). Modeling of the hysteretic metalinsulator transition in a vanadium dioxide infrared detector. Optical Engineering, 41(10), 2582-2589.

Driscoll, T., Kim, H.T., Chae, B.G., Di Ventra, M., and Basov, D. (2009). Phase-transition driven memristive system. Applied physics letters, 95(4), 043503.

Griffiths, C. and Eastwood, H. (1974). Influence of stoichiometry on the metal-semiconductor transition in vanadium dioxide. Journal of Applied Physics, 45(5), 22012206.

Gupta, V., Lucarelli, G., Castro, S., Brown, T., and Ottavi, M. (2019). Perovskite based low power synaptic memristor device for neuromorphic application. In 2019 14 th International Conference on Design 8 Technology of Integrated Systems In Nanoscale Era (DTIS), 1-6. IEEE.

Jerominek, H., Picard, F., and Vincent, D. (1993). Vanadium oxide films for optical switching and detection. Optical Engineering, 32(9), 2092-2100.

Schuman, C.D., Potok, T.E., Patton, R.M., Birdwell, J.D., Dean, M.E., Rose, G.S., and Plank, J.S. (2017). A survey of neuromorphic computing and neural networks in hardware. arXiv preprint arXiv:1705.06963.

Visintin, A. (1994). Differential models of hysteresis, volume 111. Springer Science \& Business Media.

Wang, Z., Joshi, S., Savel'ev, S., Song, W., Midya, R., Li, Y., Rao, M., Yan, P., Asapu, S., Zhuo, Y., et al. (2018). Fully memristive neural networks for pattern classification with unsupervised learning. Nature Electronics, 1(2), 137.

Zylbersztejn, A. and Mott, N.F. (1975). Metal-insulator transition in vanadium dioxide. Physical Review B, 11(11), 4383. 